\title{
Photo-Driven Hydrogen Evolution by an Artificial Hydrogenase Utilizing the Biotin-Streptavidin Technology
}

\author{
Sascha G. Keller, ${ }^{a}$ Benjamin Probst, ${ }^{b}$ Tillmann Heinisch, ${ }^{a}$ Roger Alberto*,b and Thomas R. Ward*,a \\ a Department of Chemistry, University of Basel, Mattenstrasse 24a, 4002 Basel, Switzerland, thomas.ward@unibas.ch \\ b Department of Chemistry, University of Zürich, Winterthurerstrasse 190, 8057 Zürich, Switzerland, ariel@chem.uzh.ch
}

\begin{abstract}
Photocatalytic hydrogen evolution by an artificial hydrogenase based on the biotin-streptavidin technology is reported. A biotinylated cobalt pentapyridylbased hydrogen evolution catalyst (HEC) was incorporated into different mutants of streptavidin. Catalysis with $\left[\mathrm{Ru}(\mathrm{bpy})_{3}\right] \mathrm{Cl}_{2}$ as a photosensitizer (PS) and ascorbate as sacrificial electron donor (SED) at different $\mathrm{pH}$ values highlighted the impact of close lying amino acids that may act as a proton relay under the reaction conditions (Asp, Arg, Lys). In the presence of a close-lying lysine residue, both, the rates were improved, and the reaction was initiated much faster. The X-ray crystal structure of the artificial hydrogenase reveals a distance of 8.8 A between the closest lying Co-moieties. We thus suggest that the hydrogen evolution mechanism proceeds via a single Co centre. Our findings highlight that streptavidin is a versatile host protein for the assembly of artificial hydrogenases and their activity can be fine-tuned via mutagenesis.
\end{abstract}

Keywords: artificial hydrogenase $\bullet$ hydrogen evolution $\bullet$ photosensitizer $\bullet$ biotin-streptavidin

\section{Introduction}

Splitting water into $\mathrm{O}_{2}$ and $\mathrm{H}_{2}$ using sunlight as energy source offers an attractive means to address the World's growing energy needs. ${ }^{[1-4]}$ Clean energies including solar, tidal, wind- or hydroelectric-power plants are widely used, but generate electricity which is challenging to store. Storing the energy within chemical bonds and producing an energy carrier that can be liquefied or transported as a gas is desirable. There are multiple ways of producing hydrogen on an industrial scale, yet most of them rely on carbon sources, precious metal catalysts and are not energy efficient. ${ }^{[5-7]}$ Hydrogenases are natural enzymes found in a variety of bacterial and archaeal organisms, where they generate reducing equivalents in the form of $\mathrm{H}_{2}$ or oxidize $\mathrm{H}_{2}$ in reactions that require a reductant. ${ }^{[8-11]}$ Such enzymes display exquisite catalytic properties as they operate: i) without the need of a high over-potential, ii) at high reaction rates $(1,500-20,000$ turnovers per second), iii) under physiological conditions at $\mathrm{pH} 7$ and $37^{\circ} \mathrm{C}$ in water. To achieve such unrivalled characteristics, hydrogenases are highly evolved, featuring specialized channels to deliver the substrates and extract the product: proton-, electron- and dihydrogen channels ensure that the reaction does not lead to the generation of side-products. ${ }^{[12]}$ Importantly, natural hydrogenases rely on earth-abundant metals (i.e. $\mathrm{Ni}$ and $\mathrm{Fe}$ ), but require complex maturation machineries to assemble and display modest stabilities under non-physiological conditions.

Their oxygen sensitivity and production costs call for the development of artificial hydrogenases. Several artificial hydrogenases rely on the incorporation of artificial metal cofactors in host proteins (cytochrome $c$, rubredoxin, ferredoxin) or linking to a polypeptide. ${ }^{[13-26]}$ In the past decade, the biotin-streptavidin technology has found widespread use for the assembly of artificial metalloenzymes (ArM). ${ }^{[27-33]}$ Streptavidin (Sav) is a remarkably stable and versatile homotetrameric protein (4 x 159 amino acids, ca. $65 \mathrm{kDa}$ ). Each of the four monomers can bind one equivalent of biotin with a supramolecular binding affinity of ca. $10^{13} \mathrm{M}^{-1}$, thus representing one of the strongest non-covalent interactions in nature. This feature offers the possibility to anchor abiotic cofactors within a protein environment to create artificial metalloenzymes. ArMs based on the biotinSav technology have been developed to catalyze a wide variety of water compatible reactions including: olefin metathesis, Suzuki-coupling, $\mathrm{C}-\mathrm{H}$ activation or transfer hydrogenation etc. ${ }^{[30,34-37]}$
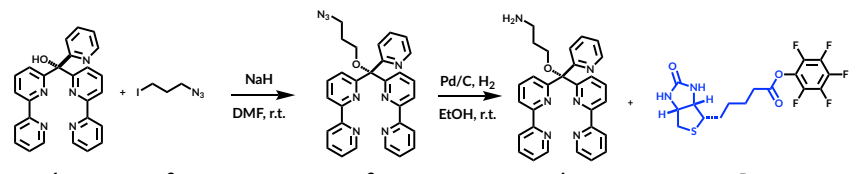

1
$\mathrm{COBr}_{2}$
$\downarrow$

3

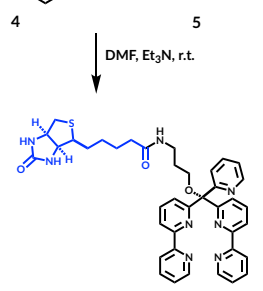

Scheme 1. Synthetic pathway of the hydrogen evolution catalyst [ $\mathrm{CoBr}(\mathrm{appy})-\mathrm{OH}] \mathrm{Br}$ and its biotinylated counterpart [ $\mathrm{CoBr}$ (appy)-Biot]Br (biotin depicted in blue, see the supporting information for details).

Herein we report on our efforts to engineer an artificial hydrogenase based on the biotin-streptavidin technology. In view of the versatility of homogeneous Co-based hydrogen-evolution catalysts, we selected this base metal for the development of $\mathrm{ArMs}$, using $\left[\mathrm{Ru}(\mathrm{bpy})_{3}\right] \mathrm{Cl}_{2}$ as photosensitizer and ascorbate as reducing agent. 


\section{Results and Discussion}

\section{Synthesis and binding studies}

The pentapyridyl (appy) ligand and the corresponding Co-catalyst [CoBr(appy)-OH]Br were selected for derivatization with a biotin anchor, Scheme ${ }^{\left[{ }^{[38]}\right.}$ The biotinylated hydrogen evolution catalyst (HEC) [CoBr(appy)-Biot]Br was synthesized in 6-steps from commercial compounds, (Scheme 1, Figures S1-S6, see SI for details). The hydroxybearing pentapyridine (appy)-OH (1) was reacted with 1-iodo-3-azidopropane (2) to afford the corresponding azido ether (appy)- $\mathbf{N}_{\mathbf{3}}(3)$. The azide group was reduced and the resulting amine (appy) $-\mathbf{N H}_{2}$ (4) was coupled to activated biotin-pentafluorophenylester (5) to afford the biotinylated ligand (appy)-Biot (6). Reaction with $\mathrm{CoBr}_{2}$ in $\mathrm{MeOH}$ yielded [ $\mathrm{CoBr}$ (appy)-Biot] $\mathrm{Br}$ as a light brown solid which was purified by recrystallization. The affinity of [CoBr(appy)-Biot]Br towards streptavidin was evaluated relying on a displacement titration using 2-(4-hydroxyphenylazo)benzoic acid (HABA). ${ }^{[39,40]}$ The titration revealed that all four biotin-binding sites of the homotetrameric Sav could accommodate one biotinylated cofactor [CoBr(appy)-Biot]Br (Figure S7). The linear segment profile of the displacement titration highlight that the affinity of [CoBr(appy)-Biot]Br for Sav WT is comparable to that of biotin itself (i.e. $>10^{10} \mathrm{M}^{-1}$ ). This demonstrates that up to four equivalents of the bulky [CoBr(appy)-Biot] $\mathrm{Br}$ cofactor can bind quantitatively to the homotetrameric Sav host.

\section{Photocatalysis and genetic optimization}

To compare the activity of the Co-precursor [ $\mathrm{CoBr}($ appy)-OH] $\mathrm{Br}$ and its biotinylated analogue [CoBr(appy)-Biot] Br, argon-flushed, buffered $(\mathrm{pH}=5)$ aqueous solutions ( $10 \mathrm{ml}$ total volume) were charged with $5 \mu \mathrm{M}$ catalyst concentration, $1 \mathrm{M} \mathrm{AscOH}$ (sacrificial electron donor), $100 \mu \mathrm{M}\left[\mathrm{Ru}(\mathrm{bpy})_{3}\right] \mathrm{Cl}_{2}$ (photosensitizer) and were irradiated with a LED (453 nm and $0.3 \mu \mathrm{E} \mathrm{s}{ }^{-1}$ ). Hydrogen evolution was continuously monitored by an automated GC as described elsewhere. ${ }^{[41]}$

With no Sav present, both [CoBr(appy)-Biot]Br and [CoBr(appy)-OH]Br display similar turnover numbers after 6.5 hours $($ TON $=1050$ and TON = 1120 after 6 hours, black and blue traces for [CoBr(appy)-Biot]Br and [CoBr(appy)-OH]Br respectively, Figure 1a). Addition of Sav WT (10 $\mu \mathrm{M}$ free biotin binding sites, corresponding to $2.5 \mu \mathrm{M}$ tetrameric Sav) did not impact the activity of $[\mathrm{CoBr}(\mathrm{appy})-\mathrm{OH}] \mathrm{Br}$, highlighting that there are no detrimental, non-specific interactions between the cofactor and Sav (grey trace, Figure 1a). In contrast, the biotinylated HEC, bound to SavWT, displayed a marked decrease in activity (TON $=820$ after 6 hours) highlighting the importance of second coordination sphere interactions on hydrogenase activity (green trace, Figure 1a).

The leveling of the activity after $3-4$ hours is owed to the accumulation of dehydroascorbic acid (DHA), the oxidized form of ascorbic acid. As shown before, addition of TCEP regenerates ascorbate, and $\mathrm{H}_{2}$ production of [CoBr(appy)-OH]Br was significantly prolonged, in many cases until full conversion of TCEP was achieved. ${ }^{[41,42]}$ a)

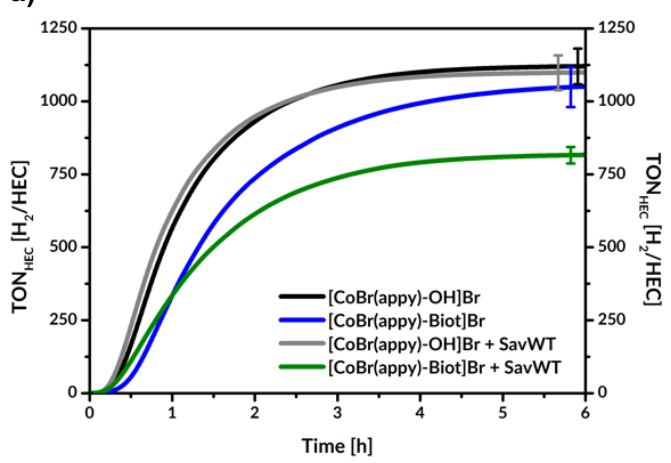

b)

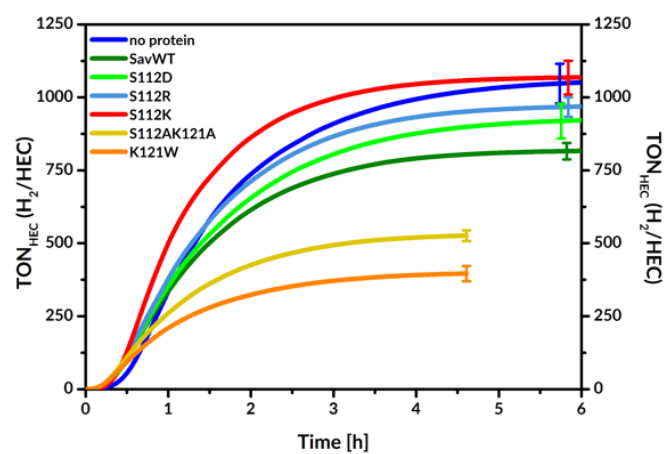

c)

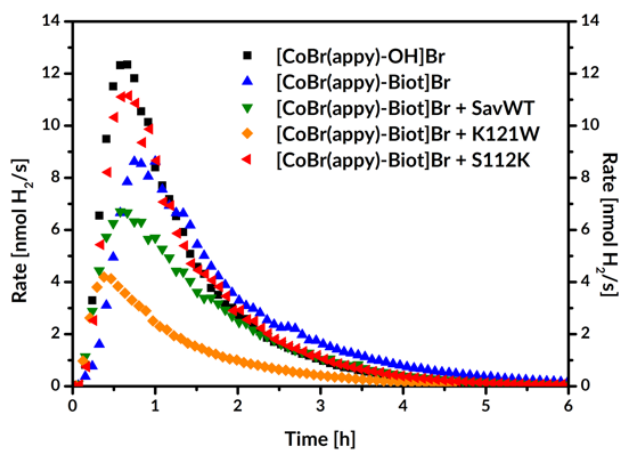

Figure 1. Summary of turnover numbers and rates of artificial hydrogenases based on the biotin-streptavidin technology. (a) hydrogen evolution profile of the parent and the biotinylated complex with and without Sav: [CoBr(appy)-OH]Br (black trace), [CoBr(appy)-OH]Br with Sav WT (grey trace), [CoBr(appy)-Biot]Br (blue trace) and [CoBr(appy)-Biot]Br with Sav WT (green trace), (b) genetic optimization of the hydrogenase activity for $[\mathrm{CoBr}(\mathrm{appy})-\mathrm{Biot}] \mathrm{Br}$ in the presence of a variety of Sav mutants: no protein (blue trace), Sav WT (green trace), Sav S112D (light green trace) Sav S112R (turquoise trace), Sav S112K (red trace), Sav S112AK121A (yellow trace) and Sav K121W (orange trace), (c) Turnover frequencies of selected hydrogenases: [CoBr(appy)-OH]Br (black trace), [CoBr(appy)-Biot]Br (blue trace) with Sav WT (green trace), Sav K121W (orange trace) and Sav S112K (red trace). Experiments were performed using $5 \mu \mathrm{M} \mathrm{HEC}, 1 \mathrm{M}$ ascorbate buffer (pH 5) and $100 \mu \mathrm{M}\left[\mathrm{Ru}(\mathrm{bpy})_{3}\right] \mathrm{Cl}_{2}, 10$ $\mu \mathrm{M}$ free biotin binding sites (when Sav was present), in $10 \mathrm{ml}$ solution and irradiation at $453 \mathrm{~nm}$ (see SI for details).

Additionally, the Sav bound catalyst should be diffusionally- and sterically hindered as compared to $[\mathrm{CoBr}(\mathrm{appy})-\mathrm{OH}] \mathrm{Br}$, thus slowing the rate of electron transfer from photogenerated, reduced photosensitizer. This, in turn, results in a higher sensitivity of the Sav system for back electron transfer to $\mathrm{DHA}$, due to a higher steady state concentration of reduced 
photosensitizer. Thus, we set out to explore the impact of the second coordination sphere of Sav. For this purpose, a focused library of Sav single point mutants was screened in combination with [CoBr(appy)Biot]Br. Building on previous studies, two close lying positions were selected: S112 and K121. Substitution of the serine residue S112 by either an Asp (Sav S112D, TON = 920 after 6 hours, light green trace, Figure 1b) or a Arg (Sav S112R, TON = 970 after 6 hours, turquoise trace, Figure 1b) lead to an increase in TON compared to Sav WT (green trace, figure 1b). We speculate that this might be due to the presence of close lying amino acids capable of acting as a proton relay, potentially facilitating outer-sphere protonation of the $\mathrm{Co}-\mathrm{H}$ species, as suggested in related studies. ${ }^{[4-48]}$ Thus an increase in the rate of $\mathrm{H}_{2}$ formation at the ArM, and a concomitant removal of reducing equivalents from the system, would be expected. That way, back electron transfer to DHA would be prevented to a certain level, in line with the observed increase in both, rate and TON. This was confirmed by the improved activity of the S112K mutant (Ser $\rightarrow$ Lys) for which the highest activity was observed (TON = 1070 after 6 hours, red trace, Figure 1b). To further investigate the critical role of acidic residues in the proximity of the $\mathrm{Co}-\mathrm{H}$ moiety, the double mutant Sav S112AK121A, in which both the naturally occurring serine and lysine at position S112 and K121 were mutated to alanine, displayed a marked decrease in activity (TON $=530$, after 4.5 hours, yellow trace, Figure 1b). Even more so, replacement of lysine by tryptophan (Sav K121W) shows an even higher erosion in activity (TON $=400$ after 4.5 hours, orange trace, Figure $1 \mathrm{~b}$ ). The reaction rates are also affected by incorporation of the cofactor into the protein. While the bare biotinylated cofactor [CoBr(appy)-Biot] $\mathrm{Br}$ has a maximum rate of $9 \mathrm{nmol} \mathrm{H}_{2} / \mathrm{s}$ (blue triangles, Figure $1 \mathrm{c})$, the non-biotinylated $\mathrm{HEC}[\mathrm{CoBr}($ appy)-OH]Br peaks at $13 \mathrm{nmol} \mathrm{H} / \mathrm{s}$ (black squares, Figure 1c) and the incorporated cofactor [CoBr(appy)-Biot]Br - Sav S112K peaks at $12 \mathrm{nmol} \mathrm{H}_{2} / \mathrm{s}$ (red triangles, Figure 1c). The presence of a proton relay in the immediate proximity of the $\{$ Co(appy)\}-moiety affects both the TON and the corresponding rates. The effect of the additional lysine is highlighted by the use of Sav WT (green triangles, Figure 1c) and the worst performing mutant $\mathrm{K} 121 \mathrm{~W}$ (orange squares, Figure 1c). In fact, since the binding pocket is symmetrical, not only two lysines are present within the binding pocket (S112K and K121) but four (2xS112K and 2xK121, Figure 5). This finding suggests that polar groups surrounding the catalytic centre can serve as proton relays and are indeed essential for its reactivity. When the polar groups are substituted by apolar groups as alanine the reactivity drops significantly.

\section{pH Dependence}

Hydrogen evolution at neutral $\mathrm{pH}$ is desirable, because it would allow the use of fresh or even sea water. ${ }^{[49]}$ Since the protein provides a second coordination sphere around the catalytic centre and lysines in the close proximity of the cofactor have a positive effect on catalytic performance, we anticipated a marked $\mathrm{pH}$ influence on catalysis.

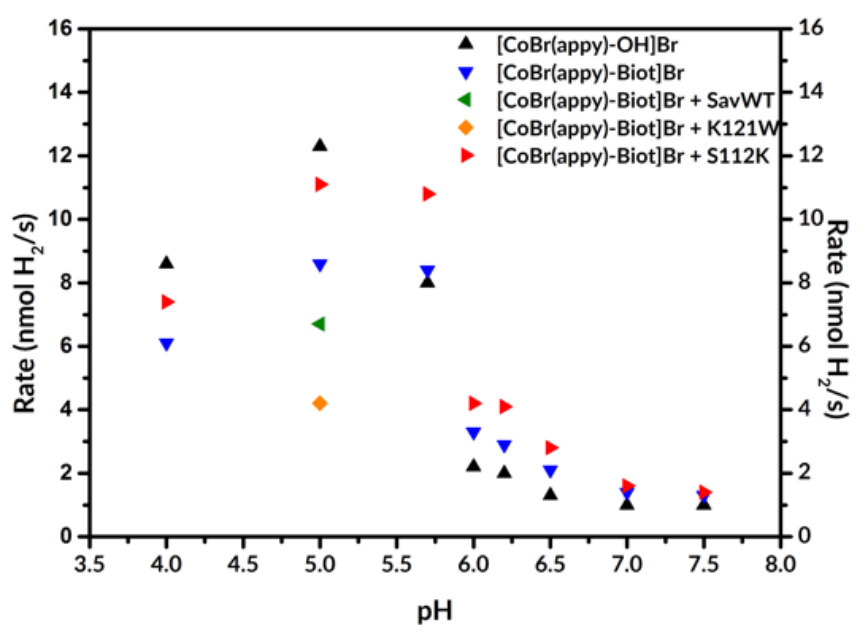

Figure 2. Maximum hydrogen production rates determined as a function of $\mathrm{pH}$ for [CoBr(appy)-OH]Br (black triangles) and [CoBr(appy)-Biot]Br - Sav S112K (red triangles), [CoBr(appy)-Biot]Br • Sav WT (green triangles), [CoBr(appy)-Biot]Br • Sav $\mathrm{K} 121 \mathrm{~W}$ (orange squares) and [CoBr(appy)-Biot] Br (blue triangles). The $\mathrm{pH}$ values were screened in buffered aqueous solutions (see SI for details).

A pH screening of [CoBr(appy)-OH]Br, [CoBr(appy)-Biot]Br and [CoBr(appy)-Biot]Br - Sav S112K was performed (Figures S8-S13 and Tables $\mathrm{S} 1$ and S2). At pH 4 and pH 5 in $1 \mathrm{M} \mathrm{AscOH}$ buffer, [CoBr(appy)-OH]Br shows higher maximal rates than the biotinylated $\mathrm{HEC}$ [CoBr(appy)-Biot]Br either inside or outside the Sav S112K (Figure 2). Increasing the pH above 5.0, leads to an inversion in trend: at pH 5.7 (using $1 \mathrm{M}$ acetate buffer with $0.1 \mathrm{M}$ $\mathrm{AscOH}$ ) the [CoBr(appy)-Biot] Br • Sav S112K displays the highest rates and turnover numbers. At pH values between 6 and 7.5 (using a $1 \mathrm{M}$ phosphate buffer, with $0.1 \mathrm{M} \mathrm{AscOH}$ ) the hydrogen production rates follow the following order: [CoBr(appy)-Biot]Br • Sav S112K > [CoBr(appy)-Biot]Br > [CoBr(appy)-OH]Br (Figure 2).

Incorporation of the biotinylated cofactor [CoBr(appy)-Biot] $\mathrm{Br}$ within Sav $\mathrm{S} 112 \mathrm{~K}$ affects both the reaction rate as well as the time required to reach the maximum rate (Figure 3). At pH = 7.5, [CoBr(appy)-Biot $] \mathrm{Br} \bullet$ Sav S112K reaches after 500 minutes its maximum rate, whereas for [CoBr(appy)$\mathrm{OH}] \mathrm{Br}, 1100$ minutes are required.

Monitoring both the rate and TON at $\mathrm{pH}=7.0$ reveals that $[\mathrm{CoBr}(\mathrm{appy})-$ Biot]Br - Sav S112K outperforms both [CoBr(appy)-OH]Br and [CoBr(appy)Biot]Br, Figure 4. The most striking difference, apart from the increase in rate, is the shorter time required until the maximum rate is reached. In line with the arguments from the previous section, protic residues on Sav could increase the rate of hydrogen formation. Thus, a lower steady state concentration of reduced HEC is expected, lowering the probability for deleterious back electron transfer, and increasing the hydrogen formation rate from the beginning. 


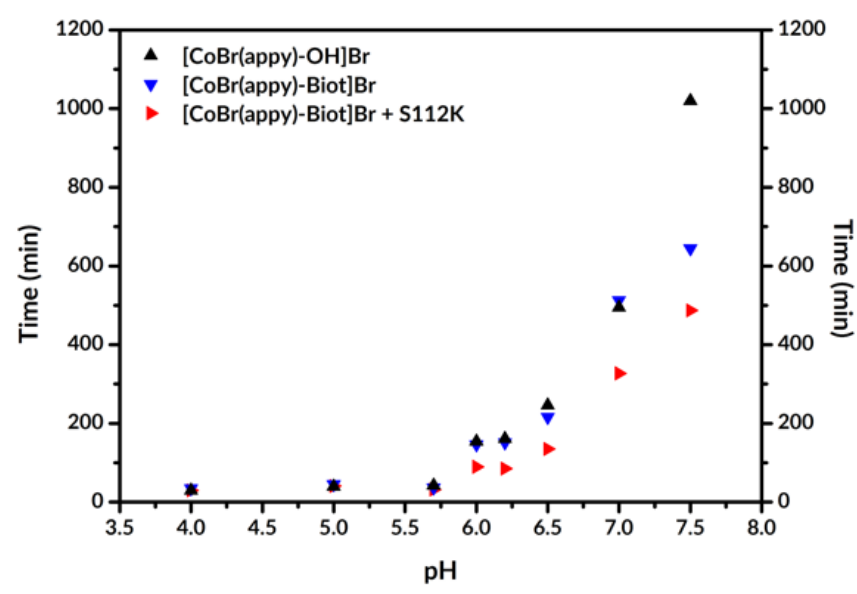

Figure 3. Summary of times required to reach the maximum rate of hydrogen production as a function of $\mathrm{pH}$. [CoBr(appy)-Biot]Br - Sav S112K (red triangles), [CoBr(appy)-OH] Br (black triangles) and [CoBr(appy)-Biot] $\mathrm{Br}$ (blue triangles).

To rule out the possibility of spurious $\mathrm{Co}$ (II) species acting as catalyst, control experiments were conducted whereby some of the catalyst's components were omitted (Figure S14). Compared to [CoBr(appy)-Biot]Br • Sav S112K, under otherwise identical reaction conditions, $\mathrm{Ru}(\mathrm{bpy})_{3}{ }^{2+}$ alone, Sav S112K, $\mathrm{CoBr}_{2}$ or $\mathrm{CoBr}_{2}$ - Sav $\mathrm{S} 112 \mathrm{~K}$ yielded only traces of hydrogen. These observations suggest that the pentapyridine-coordinated $\mathrm{Co}(\mathrm{II})$ is indeed the active species generating $\mathrm{H}_{2}$ from the Co-H moiety.

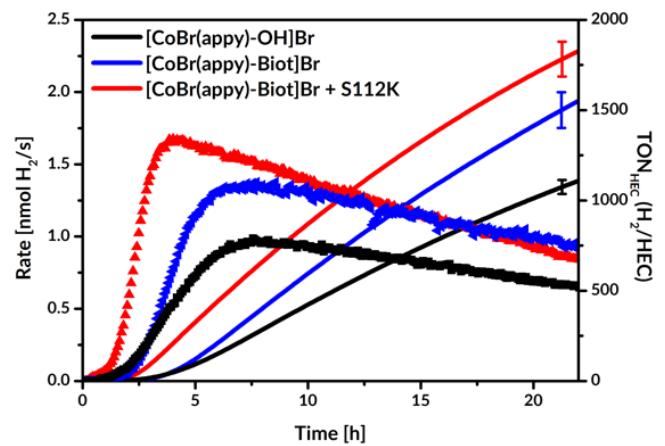

Figure 4. Comparison of the hydrogen production rate (left scale) and TON (right scale) for [CoBr(appy)-OH]Br (black trace), [CoBr(appy)-Biot]Br (blue trace) and [CoBr(appy)$\mathrm{OH}] \mathrm{Br} \bullet$ Sav S112K (red trace) upon irradiation at $453 \mathrm{~nm}$ with $100 \mu \mathrm{M}\left[\mathrm{Ru}(\mathrm{bpy})_{3}\right] \mathrm{Cl}_{2}$ in $1 \mathrm{M} \mathrm{NaHPO}_{4}$ buffer ( $\mathrm{pH} 7$ ) and $0.1 \mathrm{M} \mathrm{AscOH}$ present (See SI for details).

\section{Structural Insight}

The localization of $\mathrm{CoBr}(\mathrm{appy})$-Biot]Br within streptavidin was scrutinized by X-ray crystallography. A crystal of complex [CoBr(appy)-Biot]Br • Sav-K121A was obtained upon soaking the apoprotein crystals with an excess of cofactor overnight. The X-ray structure of [CoBr(appy)-Biot]Br • Sav S112A was solved to $1.7 \AA$ resolution (Figures 5, S15 and Table S3). Upon solving the protein structure by molecular replacement, residual electron density was visible in the biotin binding pocket. Complex $\left[\mathrm{CoH}_{2} \mathrm{O} \text { (appy)-Biot }\right]^{2+}$ was modelled in each of the four Sav monomers with full occupancy and in a single conformation. The first coordination sphere around cobalt is virtually identical to that found in the corresponding small molecule crystal structure (CCDC identifier: LILYAI). ${ }^{[38]}$

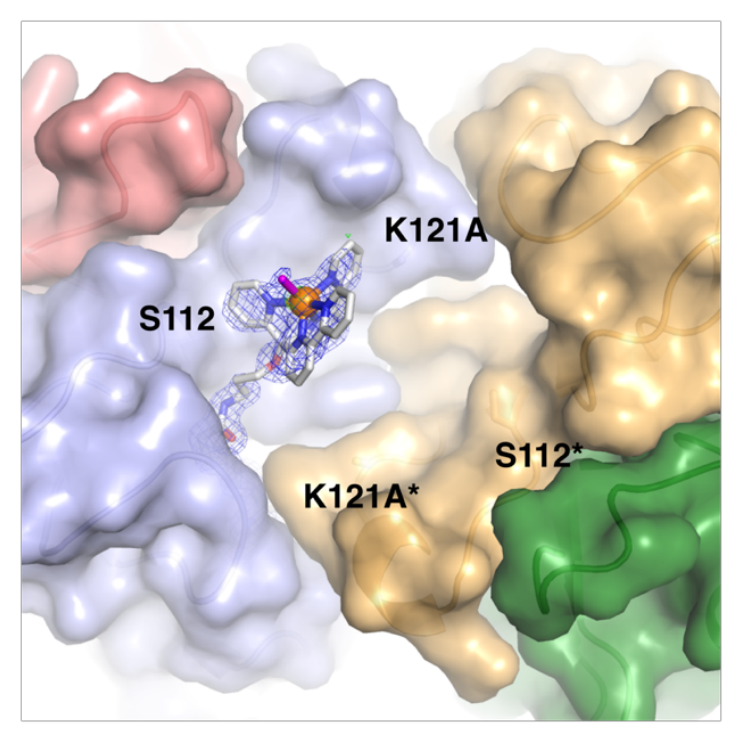

Figure 5. Close-up view of the active site in the crystal structure of complex [ $\mathrm{CoH}_{2} \mathrm{O}$ (appy)-Biot] - Sav-K121A (PDB code 6FRY). The protein is displayed as surface and cartoon model and the cofactor as stick model. The cofactor is contoured by a $2 \mathrm{Fo}-$ Fc electron density map ( $1.0 \sigma$, blue colour) and an anomalous dispersion density map (3.0 $\sigma$, green colour). The orange sphere indicates a cobalt and the magenta stick a water molecule. For clarity only one cofactor molecule is displayed per Sav tetramer.

The central metal is coordinated by the heteroaromatic groups in a distorted octahedral fashion. The axial bromide was replaced by water in the metalloenzyme. The bulky cobalt complex is accommodated within the biotin vestibule that is flanked from three side by loops 3,$4 ; 5,6$ and 7,8 , the fourth site occupying a symmetry-related cofactor molecule. The axial water ligand is exposed to the solvent and is presumed to be the substrate's binding site. The distance between two neighbouring Co-atoms is $8.8 \AA$. Considering the large distance between two catalytically competent metals, we tentatively exclude a catalytic mechanism that proceeds via a dinuclear mechanism. This hypothesis is further supported by the fact that, under typical reaction conditions, only half of the biotin binding sites are occupied with [CoBr(appy)-Biot] Br, albeit with a statistical distribution. ${ }^{[50]}$ Of the two postulated mechanisms put forward for Co-based hydrogenase mimics, ${ }^{[43,51,52]}$ we suggest that the present system operates using a single Co-site.

\section{Conclusions}

In conclusion, we have demonstrated that incorporation of a biotinylated, molecular hydrogen evolution catalyst into streptavidin mutant S112K as a protein scaffold effects its activity in a positive way. The catalyst embedded within the protein not only showed higher turnover numbers at specific $\mathrm{pH}$ values but also higher maximum rates and a shorter time to reach them in most cases. The genetically-engineered additional basic amino acid S112K close to the catalyst is beneficial for activity. We suggest that this close lying pendant base is protonated under the reaction conditions and can thus 
facilitate protonation of the $\mathrm{Co}-\mathrm{H}$ species. This hypothesis is further supported by deletion of all close-lying lysine residues. Accordingly, K121A and $\mathrm{K} 121 \mathrm{~W}$ derived hydrogenases display significantly reduced activities. Furthermore, the incorporation of the catalyst into a protein cavity prevented two Co-centres to interact with another. Since the protein embedded catalyst displayed similar or, in some cases, better activity than the free catalyst, we suggest that the reaction proceeds via a single Co centre. These findings suggest that such biohybrid catalysts may readily be optimized by genetic means. Future studies will focus on applying directed evolution schemes to improve the hydrogenase activity. Finally, we hypothesize that the host protein may be immobilized on an electrode without perturbing the immediate environment around the metal cofactor.

\section{Experimental Section}

Experimental details, synthesis procedures, spectra, tables and additional graphs can be found in the supporting information.

\section{Supplementary Material}

Supporting information for this article is available on the WWW under http://dx.doi.org/10.1002/MS-number.

\section{Acknowledgements}

SGK thanks the SNI for a PhD scholarship, TRW thanks the NCCR "Molecular Systems Engineering" and the Swiss National Science Foundation (grant 200020162348 to TRW), as well as the ERC (the DrEAM) for generous funding. BP and RA thank the University of Zürich Research Priority Project LightChEC, and the SNSF Sinergia Project CRSII2 160801/2 for funding.

\section{Author Contribution Statement}

S. G. K. performed chemistry and catalysis experiments, analyzed the data and wrote the paper. B. P. helped with the catalytic experiments, discussion of the results, and reviewed the manuscript. T. H. solved the X-Ray data and provided the related figures. R. A. and T. R. W. designed the study, supervised the work, discussed the results and corrected the manuscript. All authors have given approval to the final version of the manuscript.

\section{References}

[1] M. E. El-Khouly, E. El-Mohsnawy, S. Fukuzumi, 'Solar energy conversion: From natural to artificial photosynthesis', J. Photochem. Photobiol. C Photochem. Rev. 2017, 31, 36-83.

[2] E. S. Andreiadis, M. Chavarot-Kerlidou, M. Fontecave, V. Artero, 'Artificial Photosynthesis: From Molecular Catalysts for Light-driven Water Splitting to Photoelectrochemical Cells', Photochem. Photobiol. 2011, 87, 946-964.

[3] S. Soman, 'Molecular Systems for Solar H2 : Path to a Renewable Future', Comments Inorg. Chem. 2015, 35, 82-120.

[4] C. Liu, B. C. Colón, M. Ziesack, P. A. Silver, D. G. Nocera, 'Water splittingbiosynthetic system with $\mathrm{CO} 2$ reduction efficiencies exceeding photosynthesis', Science 2016, 352, 1210-1213.

[5] T. L. LeValley, A. R. Richard, M. Fan, 'The progress in water gas shift and steam reforming hydrogen production technologies - A review', Int. J. Hydrogen Energy 2014, 39, 16983-17000.

[6] S. E. Hosseini, M. A. Wahid, 'Hydrogen production from renewable and sustainable energy resources: Promising green energy carrier for clean development', Renew. Sustain. Energy Rev. 2016, 57, 850-866.

[7] I. Dincer, C. Acar, 'Review and evaluation of hydrogen production methods for better sustainability', Int. J. Hydrogen Energy 2015, 40, 11094-11111.

[8] W. Lubitz, H. Ogata, O. Rudiger, E. Reijerse, O. Rüdiger, E. Reijerse, 'Hydrogenases', Chem. Rev. 2014, 114, 4081-4148.

[9] F. A. Armstrong, R. M. Evans, S. V. Hexter, B. J. Murphy, M. M. Roessler, P. Wulff, 'Guiding Principles of Hydrogenase Catalysis Instigated and Clarified by Protein Film Electrochemistry', Acc. Chem. Res. 2016, 49, 884-892.

K. Pandey, S. T. A. Islam, T. Happe, F. A. Armstrong, `Frequency and potential dependence of reversible electrocatalytic hydrogen interconversion by [FeFe]-hydrogenases', Proc. Natl. Acad. Sci. 2017, 114, 3843-3848.

[11] B. J. Murphy, F. Sargent, F. A. Armstrong, 'Transforming an oxygentolerant [NiFe] uptake hydrogenase into a proficient, reversible hydrogen producer', Energy Environ. Sci. 2014, 7, 1426-1433.

P. A. Ash, R. Hidalgo, K. A. Vincent, 'Proton Transfer in the Catalytic Cycle of [NiFe] Hydrogenases: Insight from Vibrational Spectroscopy', ACS Catal. 2017, 7, 2471-2485.

[13] A. Roy, C. Madden, G. Ghirlanda, 'Photo-induced hydrogen production in a helical peptide incorporating a [FeFe] hydrogenase active site mimic', Chem. Commun. 2012, 48, 9816.

[14] M. Faiella, A. Roy, D. Sommer, G. Ghirlanda, 'De novo design of functional proteins: Toward artificial hydrogenases', Biopolymers 2013, 100, 558571.

[15] S. R. Soltau, J. Niklas, P. D. Dahlberg, K. L. Mulfort, O. G. Poluektov, L. M. Utschig, 'Charge Separation Related to Photocatalytic H2 Production from a Ru-Apoflavodoxin-Ni Biohybrid', ACS Energy Lett. 2017, 2, 230-237.

S. R. Soltau, P. D. Dahlberg, J. Niklas, O. G. Poluektov, K. L. Mulfort, L. M. Utschig, `Ru-protein-Co biohybrids designed for solar hydrogen production: understanding electron transfer pathways related to photocatalytic function', Chem. Sci. 2016, 7, 7068-7078.

[17] L. M. Utschig, S. R. Soltau, D. M. Tiede, 'Light-driven hydrogen production from Photosystem I-catalyst hybrids', Curr. Opin. Chem. Biol. 2015, 25, 1-

A. Onoda, Y. Kihara, K. Fukumoto, Y. Sano, T. Hayashi, 'Photoinduced hydrogen evolution catalyzed by a synthetic diiron dithiolate complex embedded within a protein matrix', ACS Catal. 2014, 4, 2645-2648. A. Onoda, T. Hayashi, 'Artificial hydrogenase: Biomimetic approaches controlling active molecular catalysts', Curr. Opin. Chem. Biol. 2015, 25, 133-140.

[20] Y. Sano, A. Onoda, T. Hayashi, 'Photocatalytic hydrogen evolution by a diiron hydrogenase model based on a peptide fragment of cytochrome c556 with an attached diiron carbonyl cluster and an attached ruthenium photosensitizer', J. Inorg. Biochem. 2012, 108, 159-162.

[21] S. Shima, D. Chen, T. Xu, M. D. Wodrich, T. Fujishiro, K. M. Schultz, J. Kahnt, K. Ataka, X. Hu, 'Reconstitution of [Fe]-hydrogenase using model complexes', Nat. Chem. 2015, 7, 995-1002.

T. R. Simmons, G. Berggren, M. Bacchi, M. Fontecave, V. Artero, 'Mimicking hydrogenases: From biomimetics to artificial enzymes', Coord. Chem. Rev. 2014, 270-271, 127-150. activated by synthetic components', Nature $\mathbf{2 0 1 3}, 499$, 40-41. 
J. Esselborn, C. Lambertz, A. Adamska-Venkatesh, T. Simmons, G.

Berggren, J. Noth, J. Siebel, A. Hemschemeier, V. Artero, E. Reijerse, et al., 'Spontaneous activation of [FeFe]-hydrogenases by an inorganic [2Fe] active site mimic', Nat. Chem. Biol. 2013, 9, 607-609.

J. F. Siebel, A. Adamska-Venkatesh, K. Weber, S. Rumpel, E. Reijerse, W. Lubitz, 'Hybrid [FeFe]-Hydrogenases with Modified Active Sites Show Remarkable Residual Enzymatic Activity', Biochemistry 2015, 54, 14741483.

J. W. Slater, H. S. Shafaat, 'Nickel-Substituted Rubredoxin as a Minimal Enzyme Model for Hydrogenase', J. Phys. Chem. Lett. 2015, 6, 3731-3736.

M. E. Wilson, G. M. Whitesides, 'Conversion of a Protein to a

Homogeneous Asymmetric Hydrogenation Catalyst by Site-Specific

Modification with a Diphosphinerhodium(I) Moiety', J. Am. Chem. Soc. $1978,100,306-307$.

T. Heinisch, T. R. Ward, 'Artificial Metalloenzymes Based on the BiotinStreptavidin Technology: Challenges and Opportunities', Acc. Chem. Res. 2016, 49, 1711-1721.

T. R. Ward, `Artificial metalloenzymes based on the biotin - Avidin technology: Enantioselective catalysis and beyond', Acc. Chem. Res. 2011, 44, 47-57.

F. Schwizer, Y. Okamoto, T. Heinisch, Y. Gu, M. M. Pellizzoni, V. Lebrun, R. Reuter, V. Köhler, J. C. Lewis, T. R. Ward, 'Artificial Metalloenzymes: Reaction Scope and Optimization Strategies', Chem. Rev. 2018, 118, 142231.

[31] T. K. Hyster, L. Knörr, T. R. Ward, T. Rovis, 'Biotinylated Rh(III) Complexes in Engineered Streptavidin for Accelerated Asymmetric C-H Activation', Science 2012, 338, 500-503.

[32] M. Pellizzoni, G. Facchetti, R. Gandolfi, M. Fusè, A. Contini, I. Rimoldi, 'Evaluation of Chemical Diversity of Biotinylated Chiral 1,3-Diamines as a Catalytic Moiety in Artificial Imine Reductase', ChemCatChem 2016, 8 , $1665-1670$.

[33] S. I. Mann, T. Heinisch, A. C. Weitz, M. P. Hendrich, T. R. Ward, A. S. Borovik, 'Modular Artificial Cupredoxins', J. Am. Chem. Soc. 2016, 138, 9073-9076.

[34] Y. Okamoto, T. R. Ward, in Compr. Supramol. Chem. II, Elsevier, 2017, pp. 459-510.

[35] C. Lo, M. R. Ringenberg, D. Gnandt, Y. Wilson, T. R. Ward, `Artificial metalloenzymes for olefin metathesis based on the biotin-(strept)avidin technology', Chem. Commun. 2011, 47, 12065.

[36] A. Chatterjee, H. Mallin, J. Klehr, J. Vallapurackal, A. D. Finke, L. Vera, M. Marsh, T. R. Ward, 'An enantioselective artificial Suzukiase based on the biotin-streptavidin technology', Chem. Sci. 2016, 7, 673-677.

[37] M. Dürrenberger, T. Heinisch, Y. M. Wilson, T. Rossel, E. Nogueira, L. Knörr A. Mutschler, K. Kersten, M. J. Zimbron, J. Pierron, et al., 'Artificial Transfer Hydrogenases for the Enantioselective Reduction of Cyclic Imines', Angew. Chem. Int. Ed. 2011, 50, 3026-3029.

[38] C. Bachmann, M. Guttentag, B. Spingler, R. Alberto, `3d Element Complexes of Pentadentate Bipyridine-Pyridine-Based Ligand Scaffolds: Structures and Photocatalytic Activities', Inorg. Chem. 2013, 52, 60556061.

[39] M. Wilchek, E. A. Bayer, 'Introduction to avidin-biotin technology', Methods Enzymol. 1990, 184, 5-13.

[40] M. Skander, N. Humbert, J. Collot, J. Gradinaru, G. Klein, A. Loosli, J. Sauser, A. Zocchi, F. Gilardoni, T. R. Ward, ’Artificial Metalloenzymes: (Strept)avidin as Host for Enantioselective Hydrogenation by Achiral Biotinylated Rhodium-Diphosphine Complexes', J. Am. Chem. Soc. 2004
126, 14411-14418.

[41] C. Bachmann, B. Probst, M. Guttentag, R. Alberto, 'Ascorbate as an electron relay between an irreversible electron donor and $\mathrm{Ru}(\mathrm{II})$ or $\mathrm{Re}(\mathrm{I})$ photosensitizers', Chem. Commun. 2014, 50, 6737-6739.

[42] B. Probst, M. Guttentag, A. Rodenberg, P. Hamm, R. Alberto, 'Photocatalytic $\mathrm{H} 2$ production from water with rhenium and cobalt complexes', Inorg. Chem. 2011, 50, 3404-3412.

[43] A. Rodenberg, M. Orazietti, B. Probst, C. Bachmann, R. Alberto, K. K. Baldridge, P. Hamm, 'Mechanism of Photocatalytic Hydrogen Generation by a Polypyridyl-Based Cobalt Catalyst in Aqueous Solution', Inorg. Chem. 2015, 54, 646-657.

[44] M. O'Hagan, W. J. Shaw, S. Raugei, S. Chen, J. Y. Yang, U. J. Kilgore, D. L. DuBois, R. M. Bullock, 'Moving Protons with Pendant Amines: Proton Mobility in a Nickel Catalyst for Oxidation of Hydrogen', J. Am. Chem. Soc. 2011, 133, 14301-14312.

[45] L. E. Fernandez, S. Horvath, S. Hammes-Schiffer, 'Theoretical design of molecular electrocatalysts with flexible pendant amines for hydrogen production and oxidation', J. Phys. Chem. Lett. 2013, 4, 542-546.

A. Dutta, D. L. DuBois, J. A. S. Roberts, W. J. Shaw, 'Amino acid modified Ni catalyst exhibits reversible $\mathrm{H} 2$ oxidation/production over a broad $\mathrm{pH}$ range at elevated temperatures', Proc. Natl. Acad. Sci. 2014, 111, 16286-16291.

[47] N. P. Boralugodage, R. J. Arachchige, A. Dutta, G. W. Buchko, W. J. Shaw, 'Evaluating the role of acidic, basic, and polar amino acids and dipeptides on a molecular electrocatalyst for $\mathrm{H} 2$ oxidation', Catal. Sci. Technol. 2017, 7, 1108-1121.

[48] A. Dutta, J. A. S. Roberts, W. J. Shaw, 'Arginine-containing ligands enhance H2 oxidation catalyst performance', Angew. Chem. Int. Ed. 2014, 53, 64876491.

[49] T. Shinagawa, K. Takanabe, 'New Insight into the Hydrogen Evolution Reaction under Buffered Near-Neutral pH Conditions: Enthalpy and Entropy of Activation', J. Phys. Chem. C 2016, 120, 24187-24196.

[50] A. Loosli, U. E. Rusbandi, J. Gradinaru, K. Bernauer, C. W. Schlaepfer, M. Meyer, S. Mazurek, M. Novic, T. R. Ward, ’(Strept)avidin as Host for Biotinylated Coordination Complexes: Stability, Chiral Discrimination, and Cooperativity', Inorg. Chem. 2006, 45, 660-668.

[51] S. C. Marinescu, J. R. Winkler, H. B. Gray, 'Molecular mechanisms of cobalt-catalyzed hydrogen evolution', Proc. Natl. Acad. Sci. 2012, 109, 15127-15131.

[52] S. Mandal, S. Shikano, Y. Yamada, Y. M. Lee, W. Nam, A. Llobet, S. Fukuzumi, 'Protonation equilibrium and hydrogen production by a dinuclear cobalt-hydride complex reduced by cobaltocene with trifluoroacetic acid', J. Am. Chem. Soc. 2013, 135, 15294-15297. 
